\section{Ingestão dietética de folato em gestantes do município do Rio de Janeiro}

\section{Dietary intake of folate by pregnant women of the Municipality of Rio de Janeiro}

Helaine Thomaz de Lima 1

Cláudia Saunders 2

Andréa Ramalho3

1 Secretaria de Saúde de Mesquita, Rio de Janeiro, RJ.

2 Grupo de Pesquisa em Vitamina A. Centro de Ciências da Saúde. Instituto de Nutrição da Universidade Federal do Rio de Janeiro. Av. Trompovsky, s. n. Edifício dos Institutos. Bloco J, $2^{\circ}$ andar. Cidade Universitária. Ilha do Fundão. Rio de Janeiro, RJ, Brasil. CEP: 21.944-970. E-mail: cfcoelho@osite.com.b 3 Departamento de Nutrição Social e Aplicada. Instituto de Nutrição da Universidade Federal do Rio de Janeiro.

\section{Abstract}

Objectives: to assess the folate ingestion by 201 low-risk pregnant women attended at Prenatal Service in Miguel Couto Municipal Hospital, in Rio de Janeiro.

Methods: for the dietary assessment, a survey of semi-quantitative intake frequency has been used. It was considered as adequate dietary ingestion an amount of $600 \mu \mathrm{g} /$ day or above. Additionally, maternal variables were identified by interviews and checking their medical files.

Results: the prevalence of dietary folate inadequacy was $63,7 \%$. Such a rate has occurred irrespective to maternal variables, namely, age, color, dwelling, sanitary conditions, pregnancy time, time span between deliveries, parity and nutritional conditions before pregnancy. On the other hand, the folate consume has proved to be dependent on the mothers'schooling level and the use of suplements. Less educated women have shown 2,5 times more chance (OR $=2,49$ ) of inadequate folate intake, and that not in use of suplement shown 16,3 times more chance $(O R=$ 16,30) of inadequate folate intake.

Conclusions: these findings reinforce the importance of investigating the nutritional state of folate in women at reproductive age and in pregnant women at prenatal attendance, since the nutritional deficiency at this stage of the biological cycle may be associated with undesirable obstetric results.

Key words Folic acid, Pregnancy, Diet surveys, Neural tube defects

\section{Resumo}

Objetivos: avaliar a ingestão de folato em 201 gestantes de baixo risco, atendidas no ambulatório do Serviço de Atendimento ao Pré-Natal do Hospital Municipal Miguel Couto, Rio de Janeiro.

Métodos: para avaliação dietética adotou-se o método de inquérito dietético "Freqüência de Consumo Semi-Quantitativo". Considerou-se como ingestão dietética adequada um consumo maior ou igual a $600 \mu \mathrm{g} /$ dia. Adicionalmente foram obtidas variáveis maternas através de entrevista e consulta aos prontuários.

Resultados: a prevalência de consumo dietético inadequado de folato foi de 63,7\%. Observou-se que esta ocorreu independentemente das variáveis maternas: idade materna, cor, condições de saneamento da moradia, idade gestacional, intervalo interpartal, paridade e do estado nutricional pré-gestacional. Por outro lado, o consumo de folato mostrou-se dependente do grau de escolaridade materna e do uso de suplemento. As mulheres de menor grau de escolaridade apresentaram 2,5 vezes mais chance de consumo inadequado de folato $(O R=2,49)$ e as que não relataram o uso de suplemento apresentaram 16,3 vezes mais chance de inadequação dietética de folato $(O R$ $=16,30$ ).

Conclusões: tais achados ressaltam a importância da atuação dos profissionais de saúde na investigação do estado nutricional de folato em mulheres em idade reprodutiva e em gestantes, por ocasião do acompanhamento pré-natal, pois a carência nutricional nessa fase do ciclo biológico pode estar associada com o resultado obstétrico indesejável.

Palavras-chave Ácido fólico, Gravidez, Inquéritos sobre dietas, Defeitos do tubo neural 


\section{Introdução}

O termo folato engloba o ácido fólico e outros compostos com atividade biológica similar. ${ }^{1} \mathrm{O}$ ácido fólico é requerido para o crescimento normal, na fase reprodutiva (gestação e lactação), e na formação de anti-corpos. 2 Atua como coenzima no metabolismo de aminoácidos (glicina) e síntese de purinas e pirimidinas, síntese de ácido nuclêico DNA e RNA ${ }^{3}$ e é vital para a divisão celular e síntese protêica. ${ }^{4-6}$ Conseqüentemente sua deficiência pode ocasionar alterações na síntese de DNA e alterações cromossomiais. 5

A deficiência de folato na gestação está associada com a anemia megaloblástica, causada pela produção anormal de hemácias. Alguns fatores interferem nos baixos níveis séricos de ácido fólico tais como, alimentos selecionados, armazenamento dos alimentos, perda na cocção, entre outros. ${ }^{1}$ A deficiência de folato também pode estar associada com várias complicações na gestação, tais como aborto espontâneo, síndromes hipertensivas da gravidez (pré-eclâmpsia), retardo do crescimento intra-uterino e hemorragia. ${ }^{5}$ Scholl et al. ${ }^{7}$ observaram que mulheres com baixa ingestão de folato e níveis séricos de folato inadequados na $28^{\mathrm{a}}$ semana gestacional, apresentaram maior risco de parto prematuro e de conceberem bebês de baixo peso ao nascer .

Evidências epidemiológicas, clínicas e teratológicas têm demonstrado que o folato está envolvido na prevenção e patogênese dos defeitos do tubo neural (DTN). 8-11 Sabe-se que as gestantes são propensas a desenvolverem deficiência de folato provavelmente devido ao aumento da demanda desse nutriente para o crescimento fetal e dos tecido maternos. Outros fatores que contribuem para a deficiência de folato são a dieta inadequada, hemodiluição fisiológica gestacional e influências hormonais. 11,12

As anomalias descritas mais freqüentemente são anencefalia e espinha bífida, que são acompanhadas de mau prognóstico.1,3 A anencefalia é uma anomalia grave, importante causa de morte perinatal e mais freqüentemente observada na Grã-Bretanha.1,13 Os portadores de espinha bífida, defeito na coluna vertebral, produzida por fissuras que envolvem defeitos vertebrais e neurais associados, crescem com paralisia nos membros inferiores e graus variados de incontinência intestinal e de bexiga.13 Os sobreviventes geralmente requerem cuidados médicos cirúrgicos complexos. 13,14

A prevalência dos DTN é de $0,1 \%$ em todas as gestações e o risco de recorrência é de 2 a 5\%.1 Por outro lado, os DTN podem ocorrer em $95 \%$ dos casos em casais considerados de baixo risco, isto é, sem história de má-formações congênitas em gestações anteriores. ${ }^{1}$

A prevenção dos DTN tem sido considerada de grande importância para a saúde pública em vista da necessidade de cuidados especializados para o tratamento, aumentando os custos para a família e para os países. Face ao exposto, é de grande importância a avaliação do consumo de folato no período gestacional, bem como a identificação das variáveis maternas de maior associação com o consumo inadequado do micronutriente, visando fornecer subsídios para elaboração de medidas de intervenção à serem implementadas pelo profissional de saúde que atue na assistência pré-natal, na tentativa de melhorar a ingestão de folato e minimizar os riscos associados com o consumo inadequado.

\section{Métodos}

Trata-se de um estudo descritivo do tipo transversal, 15 no qual estudaram-se 201 gestantes de baixo risco, sendo excluídas as portadoras de diabetes gestacional, síndromes hipertensivas da gravidez, hepatopatias, cardiopatias ou outras patologias com o início prévio ao período gestacional, e as de gestação gemelar, atendidas no ambulatório do Serviço de Atendimento ao Pré-Natal do Hospital Municipal Miguel Couto, Rio de Janeiro, RJ, Brasil. A coleta de dados ocorreu no período de abril a novembro de 1993, após aprovação pelo comitê de ética do referido Hospital e pela Secretaria Municipal de Saúde.

A coleta de dados ocorreu após o consentimento escrito da gestante, e foi realizada através de entrevista por pesquisadores treinados e reciclados periodicamente. O instrumento para a coleta de dados foi prétestado $(n=20)$ antes da elaboração final.

As variáveis maternas foram obtidas através de entrevista e consulta aos prontuários. As condições de saneamento foram classificadas em adequadas quando estavam presentes na residência os serviços abastecimento de água de rede pública com canalização interna, esgoto ligado à rede pública e coleta de lixo; e inadequadas quando um dos serviços estava ausente. Quanto à história reprodutiva, considerouse como primeiro trimestre da gestação quando a idade gestacional foi de até a $13^{\mathrm{a}}$ semana, segundo trimestre entre $14^{\mathrm{a}}$ e $27^{\mathrm{a}}$ semana e terceiro trimestre acima da $28^{\mathrm{a}}$ semana gestacional. $16 \mathrm{O}$ intervalo interpartal foi considerado adequado quando o tempo entre o último parto e o parto atual foi maior ou igual a 24 meses. 16

Para avaliação dietética adotou-se o método de inquérito dietético "Freqüência de Consumo Semi- 
Quantitativo",17 composto por uma lista de alimentos previamente selecionados no pré-teste do instrumento em subamostra $(n=20)$ de gestantes, que apresentavam as mesmas características das mulheres incluídas no estudo. Investigou-se a freqüência do consumo dos alimentos, conforme as categorias de consumo diária, semanal, quinzenal e mensal. A quantificação dos alimentos foi realizada em medidas caseiras ou em porções. $\mathrm{O}$ teor de folato nos alimentos foi obtido em $\mu \mathrm{g} .18$ após a transformação das porções dos alimentos em gramas, 19 a partir de todas as categorias de consumo (número de vezes por dia, semana, quinzena, mês) dividindo-se pelo número de dias da categoria da freqüência. Considerou-se $600 \mu \mathrm{g} / \mathrm{dia}$ como ingestão dietética adequada, segundo a recomendação do Institute of Medicine. 12

Para a avaliação antropométrica pré-gestacional das entrevistadas, obteve-se o índice de massa corporal (IMC) pré-gestacional e classificou-se o estado nutricional segundo as recomendações do Institute of Medicine 20 e as propostas pelo Sistema de Vigilância Alimentar e Nutricional (SISVAN) para aplicação nas gestantes brasileiras. ${ }^{21}$

$\mathrm{Na}$ análise estatística foi empregado o teste Quiquadrado, considerando-se as variáveis categorizadas em: dependente dicotomizada - consumo adequado e inadequado de folato; e as variáveis independentes - idade materna - $<20$ anos, de 20 a 34 e maior ou igual a 35 anos; cor - branca e não branca; condição de saneamento da moradia adequada e inadequada; idade gestacional - primeiro, segundo e terceiro trimestre; intervalo interpartal adequado e inadequado; estado nutricional antropométrico normal ou desvio ponderal, segundo o IMC pré-gestacional; paridade - nulípara, de um a três partos e maior que três partos; e escolaridade materna primeiro grau incompleto e de primeiro grau completo a nível superior. Adotou-se também o teste $t$ de Student para a comparação de médias e o nível de significância estabelecido foi de $5 \%$.

\section{Resultados}

Segundo as características sócio-demográficas e antropométricas da amostra estudada (Tabela 1), observou-se que a maior parte dessas $(66,2 \%)$ eram adultas (20 a 34 anos), de cor branca $(59,7 \%)$, e em relação ao grau de escolaridade verificou-se que quase $80 \%$ das gestantes eram de baixa escolaridade. $\mathrm{Na}$ avaliação do estado nutricional pré-gestacional, verificou-se que $52,4 \%$ das gestantes apresentaram IMC pré-gestacional normal, enquanto que $47,6 \%$ apresentaram desvio ponderal pré-gestacional. As condições de saneamento das moradias foram predominantemente classificadas como adequadas, já que grande proporção das residências $(73,6 \%)$ tinha água e esgoto ligado a rede pública e coleta de lixo regular. 
Características sócio-demográficas e antropométricas de 201 gestantes avaliadas no Serviço de Pré-Natal no Hospital Municipal Miguel Couto. Rio de Janeiro, RJ, Brasil.

\begin{tabular}{|c|c|c|}
\hline Características & $\mathbf{n}$ & $\%$ \\
\hline \multicolumn{3}{|l|}{ Idade materna (anos) } \\
\hline$<20$ & 42 & 20,8 \\
\hline 20 a 34 & 133 & 66,2 \\
\hline$\geq 35$ & 26 & 12,9 \\
\hline Total & 201 & 100,0 \\
\hline \multicolumn{3}{|l|}{ Cor } \\
\hline Branca & 120 & 59,7 \\
\hline Negra, mulata ou parda & 81 & 40,3 \\
\hline Total & 201 & 100,0 \\
\hline \multicolumn{3}{|l|}{ Escolaridade } \\
\hline Analfabeta a primeiro grau incompleto & 158 & 78,6 \\
\hline Primeiro grau completo a superior & 33 & 16,4 \\
\hline Total & 201 & 100,0 \\
\hline \multicolumn{3}{|l|}{ IMC Pré-gestacional } \\
\hline Baixo peso & 43 & 23,0 \\
\hline Normal & 98 & 52,4 \\
\hline Sobrepeso & 38 & 20,3 \\
\hline Obesa & 8 & 4,3 \\
\hline Total & 187 & 100,0 \\
\hline \multicolumn{3}{|l|}{ Condições de saneamento da moradia } \\
\hline Inadequada & 53 & 26,4 \\
\hline Adequada & 148 & 73,6 \\
\hline Total & 201 & 100,0 \\
\hline
\end{tabular}

Em relação às características obstétricas das gestantes, verificou-se que concernente à idade gestacional, a maioria $(50,3 \%)$ das entrevistadas estava no segundo trimestre gestacional, e que $18,4 \%$ e $31,3 \%$ se encontravam no primeiro e terceiro trimestre da gestação, respectivamente. Mais da metade da amostra $(64,7 \%)$ apresentou intervalo interpartal inadequado. Já em relação à paridade, 41,8\% eram nulí- paras e $68,2 \%$ apresentavam história de mais de um parto.

A inadequação do consumo de folato dietético foi de $63,7 \%$ (Figura 1). E observou-se que esta ocorreu independentemente $(\mathrm{p}>0,05)$ das variáveis maternas: idade materna, cor, condições de saneamento da moradia, idade gestacional, intervalo interpartal, paridade e estado nutricional pré-gestacional. 


\section{Figura 1}

Ingestão dietética de folato de 201 gestantes avaliadas no Serviço de Pré-Natal no Hospital Miguel Couto. Rio de Janeiro, RJ, Brasil.

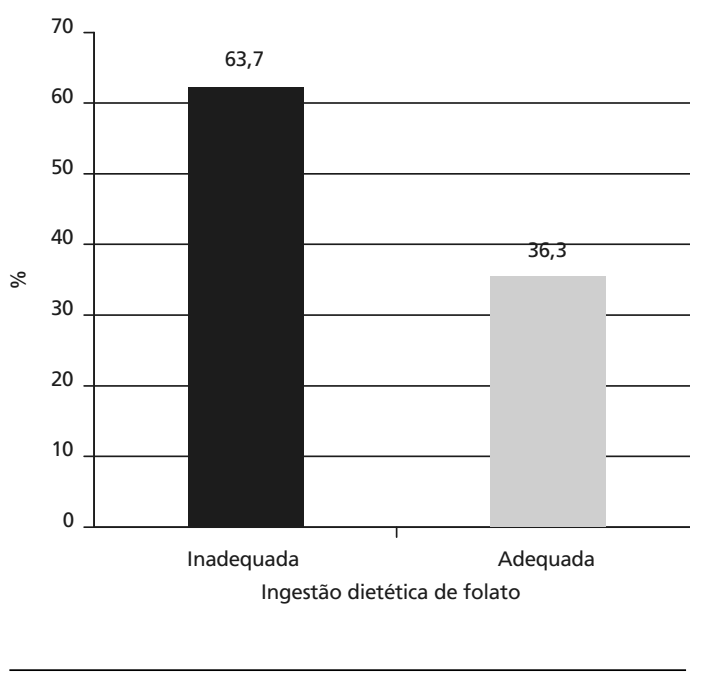

Por outro lado, a ingestão de folato mostrou ser dependente do grau de escolaridade materna (Tabela $2)$. As mulheres de menor grau de escolaridade apresentaram 2,5 vezes mais chance de ingestão inadequada de folato em comparação com as de maior escolaridade ( $O R=2,49)$. Tal associação foi confirmada na análise das médias de ingestão dietética, que para o grupo de baixa escolaridade foi de $714,5 \mu \mathrm{g}$ e para o grupo de maior escolaridade foi de $1.162,9 \mu \mathrm{g}$ $(\mathrm{t}=2,909 ; \mathrm{p}=0,005)$

$\mathrm{Na}$ Tabela 3, verifica-se a associação entre o uso da suplementação e a ingestão dietética do folato. As gestantes que não relataram o uso de suplemento vitamínico contento folato, apresentaram 16 vezes mais chance de ingestão inadequada de folato em comparação as que fizeram a suplementação (OR = 16,3). Tal associação foi confirmada pelas médias de ingestão dietética de folato, que para o grupo de mães não suplementadas foi de 400,2 + 323,1 $\mu \mathrm{g}$ e para as suplementadas foi de $1.529,8 \pm 885,8 \mu \mathrm{g}(\mathrm{t}=$ $10,504 ; \mathrm{p}<0,001)$

Associação entre o grau de escolaridade materna e a ingestão de folato de 201 gestantes avaliadas no Serviço de Pré-Natal no Hospital Miguel Couto. Rio de Janeiro, RJ, Brasil.

\begin{tabular}{|c|c|c|c|c|c|c|c|}
\hline \multirow[t]{3}{*}{ Grau de escolaridade materna } & \multicolumn{4}{|c|}{ Ingestão de folato } & & & \\
\hline & \multicolumn{2}{|c|}{ Inadequada } & \multicolumn{2}{|c|}{ Adequada } & \multirow[b]{2}{*}{ Total } & \multirow[b]{2}{*}{ OR } & \multirow[b]{2}{*}{$\mathrm{IC} 95 \%$} \\
\hline & $\mathrm{n}$ & $\%$ & $n$ & $\%$ & & & \\
\hline \multirow[t]{2}{*}{ Analfabeto +primeiro grau incompleto } & 108 & 68,4 & 50 & 31,6 & 158 & & \\
\hline & & & & & & 2,49 & $(1,25-4,94)$ \\
\hline Primeiro grau completo a superior & 20 & 46,5 & 23 & 53,5 & 43 & & \\
\hline Total & 128 & 63,7 & 73 & 36,3 & 201 & & \\
\hline
\end{tabular}

$\chi^{2}=6,97, g l=1, p=<0,008$ 
Associação entre o uso de suplemento vitamínico-mineral contendo folato e ingestão dietética de folato de 201 gestantes avaliadas no Serviço de Pré-natal no Hospital Miguel Couto. Rio de Janeiro, RJ, Brasil.

\begin{tabular}{|c|c|c|c|c|c|c|c|}
\hline \multirow[t]{3}{*}{ Suplementação contendo folato } & \multicolumn{4}{|c|}{ Ingestão de folato } & & & \\
\hline & \multicolumn{2}{|c|}{ Inadequada } & \multicolumn{2}{|c|}{ Adequada } & \multirow[b]{2}{*}{ Total } & \multirow[b]{2}{*}{ OR } & \multirow[b]{2}{*}{$(\mathrm{IC} 95 \%)$} \\
\hline & $\mathrm{n}$ & $\%$ & $\mathrm{n}$ & $\%$ & & & \\
\hline \multirow[t]{2}{*}{ Não } & 109 & 85,2 & 19 & 14,8 & 128 & & \\
\hline & & & & & & 16,30 & $(7,97-33,32)$ \\
\hline Sim & 19 & 26,0 & 54 & 74,0 & 73 & & \\
\hline Total & 128 & 63,7 & 73 & 36,3 & 201 & & \\
\hline
\end{tabular}

$\chi^{2}=70,274, g l=1, p<0,00$

\section{Discussão}

No presente estudo observou-se alta prevalência de consumo dietético inadequado de folato, sendo que as gestantes de baixa escolaridade e as que não usam suplementos contendo folato foram as de maior risco, merecendo essas destaque na assistência prénatal. Com isso, as gestantes e os conceptos estão mais expostos às consequiências da carência nutricional que tem graves repercussões ao processo reprodutivo.

Um aporte satisfatório de folato antes da concepção e durante a gestação se reveste em grande importância, visando garantir um suprimento adequado ao feto, já que baixa reserva orgânica materna pode acarretar sérios danos para o crescimento e desenvolvimento infantil, podendo elevar os índices de morbimortalidade do grupo.

Alguns pesquisadores reconhecem que a ingestão adequada de macro e micro nutrientes durante a gestação deve ser vista como condição fundamental para a saúde do concepto e redução das desordens congênitas.22-24 A deficiência de folato durante a gestação está associada com anemia megaloblástica devido ao defeito na maturação no núcleo das hemácias, mas o mecanismo ainda não é totalmente esclarecido. 3,25 Outros sinais e sintomas da carência do folato são: diarréia, queilose e glossite. As manifestações hematológicas incluem macrocitose, baixa concentração de reticulócitos, baixa contagem de leucócitos e plaquetas, anicitose e hipersegmentação dos núcleos dos neutrófilos. ${ }^{3,18,25} \mathrm{E}$ também estão associados com a deficiência de folato: a irritabilidade, anorexia, perda de peso, cefaléia, dispnéia, palpitação e ainda níveis sangüíneos elevados de homocisteína. 25,26

Além disso, a deficiência de folato tem sido reconhecida como um dos fatores associados ao desenvolvimento dos defeitos de tubo neural (DTN). Os achados descritos em relação ao papel do folato no processo reprodutivo, sugerem que o estoque corporal adequado desta vitamina no período periconcepção e no período crítico de desenvolvimento embrionário (da quarta a oitava semana) está associado com a redução de má-formações do sistema nervoso central, principalmente de DTN, abortos espontâneos e baixo peso ao nascer, contribuindo, dessa forma, para o adequado desenvolvimento fetal. 8,11

Dentre os DTN os mais prevalentes são a espinha bífida e a anencefalia, representando um sério problema para o setor saúde e para a família dos conceptos, sendo a sua prevenção possível na maioria dos casos. ${ }^{1}$ A espinha bífida é o mais comum caso de paralisia e os programas de intervenção tem reduzido de nove para seis os casos de defeitos de nascimento associado com DTN. 25

O papel do folato na prevenção dos DTN, está associado com sua função de prover uma unidade de carbono para a síntese de ácido nucléicos (ácidos desoxirribonucléico - DNA e ribonucléico - RNA). O DNA é responsável pela informação genética, enquanto que o RNA está envolvido no processo de tradução do DNA em proteína. 25,26 Com isso, o folato em associação com a vitamina B12 é indispensável 
para a síntese do DNA. A deficiência do folato afeta a divisão celular e afeta a síntese de nucleotídeos (purina e pirimidina), acarretando numa diminuição na síntese de DNA e conseqüentemente causando má-formação do sistema nervoso central e DTN.27-29 Durante a formação do sistema nervoso central, ocorrem processos chamados de neurulação primária e secundária e, qualquer imperfeição durante esses processos no período entre a quarta e a oitava semana de gestação, expõe o feto aos riscos de aparecimento de DTN. $13,14,24,30$

As alterações na utilização do folato durante a gestação também podem estar associadas com fatores hormonais esteróides. $24,31 \mathrm{O}$ folato dietético é convertido através de uma série de reações de redução para sua atuação como coenzima no fígado. Como a desativação dos hormônios progesterona e estrogênio também ocorre no fígado, antes de sua excreção, através de mecanismos semelhantes aos de ativação do folato, acredita-se que os altos níveis de esteróides podem interferir no metabolismo normal do ácido fólico. É descrito na literatura que algumas mulheres em uso de contraceptivos à base de esteróides desenvolvem deficiência de folato reforçando a possível interrelação entre esse nutriente e os hormônios esteróides. 3,32,14

Em face do exposto, na década de 80, o United Kingston Medical Research Council, 25 iniciou um estudo multicêntrico, com mulheres com história de DTN na prole, visando avaliar o impacto da suplementação com $400 \mu \mathrm{g}$ de ácido fólico na sua recorrência. Foi observada uma redução no risco de recorrência de $71 \%$. Outros estudos foram realizados e os resultados também confirmaram a redução significativa na prevenção dos DTN em vários países. 1,27,2830

O mecanismo de prevenção dos DTN pelo uso do ácido fólico ainda não é totalmente conhecido. As evidências epidemiológicas e bioquímicas sugerem que o problema primário não esteja relacionado com a falta de folato na dieta, mas sim com a sua reabsorção e/ou metabolismo. Por outro lado, existe durante a gestação, um aumento do metabolismo do folato, necessitando a gestante de um adicional de 200 a $300 \mu \mathrm{g} / \mathrm{dia}$. A concentração sérica de folato cai de $6 \mathrm{ng} / \mathrm{ml}$ no estado pré-gravídico para $4,5 \mathrm{ng} / \mathrm{ml} \mathrm{du}$ rante a gestação. Assim a suplementação com doses elevadas de ácido fólico, aumentam sua concentração nos fluidos teciduais, corrigindo a deficiência local nos mesmos. 1

A suplementação de folato através de alimentos fortificados e/ou suplementados, tem sido recomendada no período pré-concepção por um mês e pósconcepção por dois meses, ou seja, período no qual termina o período crítico do desenvolvimento do sistema nervoso central. ${ }^{1}$ Ainda não há consenso se a suplementação de ácido fólico deva ser associada com outros nutrientes e a quantidade exata a ser administrada. O Center of Diseases Control (CDC) 1 tem recomendado a dose diária de $4 \mathrm{mg} /$ dia para gestantes consideradas de alto risco para DTN. A Food and Drug Administration (FDA) segundo Cha ${ }^{1}$ também tem recomendado a utilização de folato na ordem de $400 \mu \mathrm{g} / \mathrm{dia}$ em todas as mulheres em idade fértil, visando reduzir a incidência de DTN. Para atingir a dose necessária, recomenda-se a utilização de alimentos fonte, alimentos fortificados ou a utilização de suplemento medicamentoso. $2,33,34 \mathrm{Cha},{ }_{1}$ recomenda que todas as mulheres em idade reprodutiva, independente de pertencerem ao grupo de risco para DTN, recebam suplementação de ácido fólico, especialmente durante a gestação, cuja dose deve ser de $400 \mu \mathrm{g} / \mathrm{dia}$ até a oitava semana gestacional. A suplementação deve ser feita fora do horário das refeições, objetivando maior taxa de absorção. Associado à suplementação deve-se estimular o consumo de dieta equilibrada e rica em alimentos fonte de ácido fólico, ${ }^{1}$ tais como levedo de cerveja, fígado de boi, espinafre cozido, feijão branco e vermelho cozido, brócolis, germe de trigo, suco de laranja, repolho cru.1,3 Por outro lado, a suplementação deve ser planejada com cautela, pois, é relatado que doses acima de $350 \mu \mathrm{g} / \mathrm{dia}$ reduz absorção de zinco ${ }^{3,18}$ e a maioria dos suplementos de folato utilizados no prénatal excedem a ingestão recomendada (ingestão dietética de referência - IDR). 12

O Institute of Medicine 12,18 reconhece as dificuldades na avaliação do consumo de folato, incluindo a limitação dos métodos de inquérito dietético que podem superestimar ou subestimar a ingestão dos alimentos; dificuldades na identificação dos alimentos fonte associada com a limitada disponibilidade de alimentos fonte incluídos nas tabelas de composição química; além disso podemos citar a falta de dados sobre alimentos fonte de folato produzidos e cultivados no Brasil. Estas limitações também têm sido descritas para outros nutrientes tais como vitamina A. 35

\section{Conclusões}

Tendo em vista a elevada prevalência de ingestão dietética inadequada de folato dietético no grupo estudado, ressalta-se a importância da atuação dos profissionais de saúde na investigação do estado nutricional de folato em mulheres em idade reprodutiva e, em gestantes, por ocasião do acompanhamento 
pré-natal. A carência nutricional nessa fase do ciclo biológico pode estar associada com o resultado obstétrico indesejável. Por outro lado, as medidas de intervenção devem ser planejadas de forma que a sua abrangência contemple as atividades desenvolvi- das nas unidades de saúde e, em todos os locais onde as usuárias buscam ações de atenção primária de saúde, visando a melhoria dos níveis de ingestão e de reservas maternas, que pode contribuir para o desenvolvimento normal do feto.

\section{Referências}

1. Cha SC. Prevenção dos defeitos do tubo neural (DTN). Rev Soc Bras Med Fetal 1996; 1: 7-11.

2. Rose NC, Mennuti MT. Periconceptional folate supplementation and neural tube defects. Clin Obstet Gynecol 1994; 37: 605-20.

3. Worthington-Roberts BS, Williams SR. Nutrition in pregnancy and lactation. Dubuque: Brown \& Benchmark; 1997.

4. Luke B. Maternal-fetal nutrition. Clin Obstet Gynecol 1994; 37: 93-109.

5. Ramakrishnan V, Manjrekar R, Rivera J, Gonzáles-Cossio T, Martorell R. Micronutrients and pregnancy outcome: a review of the literature. Nutr Res 1999; 19: 103-59.

6. Rondó PHC, Abbott R, Rodrigues LC, Tomkins AM. Vitamin A, folate, and iron concentrations in cord and maternal blood of intra-uterine growth retarded and appropriate birth weight babies. Eur J Clin Nutr1995; 49: 391-99

7. Scholl TO, Hediger ML, Scholl JI, Khoo CS, Fischer RL. Dietary and serum folate: their influence on the outcome of pregnancy. Am J Clin Nutr 1996; 63: 520-5.

8. American Academy of Pediatrics. Committee on Genetic. Folic acid for the prevention of neural tube defects. Pediatrics 1993; 92: 493-4.

9. Locksmith GJ, Duff P. Preventing neural tube defects: the importance of periconceptal folic acid supplements. Obstet Gynecol 1998; 91: 1027-34.

10. Schorah CJ, Habibzadeh N, Wild J, Smithells RW. Possible abnormalities of folate and vitamin B12 metabolism associated with neural tube defects. Ann NY Acad Sci 1993; 678: 81-91.

11. Steegers-Theunissen RP. Folate metabolism and neural tube defects: a review. Eur J Obstet Gynecol Reprod Biol 1995; 61: 39-48.

12. Institute of Medicine. Food and Nutrition Board. Dietary reference intakes for thiamin, riboflavin, niacin, vitamin B6, folate, vitamin B12, pantothenic acid, biotin, and choline. Washington, DC: National Academy; 2000.

13. Moore KL, Persaud TVN. Embriologia básica. Rio de Janeiro: Guanabara Koogan; 1995.

14. Yaslle M. Nutrição na gestação e lactação. In: Dutra-deOliveira JE, Marchini JS. Ciências nutricionais. São Paulo: Savier; 1998. p. 167-77.

15. Hennekens $\mathrm{CH}$, Buring JE. Epidemiology in medicine. Boston: Little Brown; 1987.

16. Ministério da Saúde. Assistência pré-natal: manual técnico. Brasília, DF: Ministério da Saúde; 2000.
17. Bower C, Fiona JS, Darrly JN. Maternal folate status and the risk for neural tube defects. Ann NY Acad Sci 1993; 678: 148-55.

18. United States. Department of Agriculture (USDA). Composition of foods. Washington, DC: USDA; 1976/1986. (Handbook, 8)

19. Pinheiro ABV, Lacerda EMA, Benzecry EH, Gomews MCS, Costa VM. Tabela para avaliação de consumo alimentar em medidas caseiras. São Paulo: Atheneu; 2001.

20. Institute of Medicine. Nutrition during pregnancy and lactation: an implementation guide. Washington, DC: National Academy; 1992

21. Engstrom EM, Oliveira ES, Barros DC, Zaborowski EL, Monteiro KA. Diagnóstico nutricional de gestantes. In SISVAN (Sistema de Vigilância Alimentar e Nutricional). Instrumento para o combate aos distúrbios nutricionais em serviços de saúde: o diagnóstico nutricional. Rio de Janeiro: Fundação Oswaldo Cruz (FIOCRUZ)) 2002. p. 61-84.

22. Coutsouis A, Adhikari M, Coovadia HM. Serum vitamin A (retinol) concentrations and association with respiratory disease in premature infants. J Trop Pediatr 1995; 44 : 230-3.

23. Underwood BA. Maternal vitamin A status and its importance in infancy and early childhood. Am J Clin Nutr 1994; 59 Suppl: 517S-24S

24. Rosso P. Physiological changes of pregnancy. In: Rosso P. Nutrition and metabolism in pregancy. Mother and fetus. New York: Oxford University; 1990. p. 3-40.

25. MRC (Medical Research Council). Vitamin Study Research Group. Prevention of neural tube defects. Lancet 1991; 338: 131-7.

26. Scholl OT, Johnson WG. Folic acid: influence on the outcome of pregnancy. Am J Clin Nutr 2000; 71 Suppl: 1295S-1303S.

27. Bower C, Stanley FJ. Dietary folate as a risk factor for neural tube defects: evidence from a case-control study in Western Australia. Med J Aust 1989; 150: 613-9.

28. Czeilel AE. Folic acid in the prevention of neural tube defects. Pediatr Gastroenterol Nutr 1995; 20: 4-16.

29. Czeilel AE, Dudás SI. Prevention of the first ocorrence of neural-tube defects by periconceptional vitamin supplementation. N Eng J Med 1992; 327: 1832-5.

30. Werler BM, Shapiro S, Mitchell AA. Periconceptional folic acid exposure and risk of current neural tube defects. JAMA 1993; 269: 1257-61. 
31. Vannucchi H, Jordão Júnior AA. Vitaminas hidrossolúveis. In: Dutra-de-Oliveira JE, Marchini JS. Ciências nutricionais. São Paulo: Sarvier; 1998. p.191-207.

32. Linder MC. Nutritional biochemistry and metabolism - with clinical applications. 2. ed. New Jersey: Prentice Hall International; 1991

33. Bailey BL. New standard for dietary folate intake in pregnant women. Am J Clin Nutr 2000; 71 Suppl: 1304S-7S
34. Lewis CJ, Crane NT, Wilson DB, Yetley EA. Estimated folate intakes: data updated to reflect food fortificatication, increased bioavailability, and dietary supplement use. Am J Clin Nutr 1999; 70: 198-207.

35. Saunders C, Ramalho RA, Accioly E, Paiva F. Utilização das tabelas de composição dos alimentos na avaliação do risco de hipovitaminose A. Arch Latinoam Nutr 2000; 50: $237-42$.

Recebido para publicação em 5 de maio de 2002 Versão final reapresentada em 2 de julho de 2002 Aprovado em 4 de agosto de 2002 